\title{
Tsafon
}

Revue d'études juives du Nord

$77 \mid 2019$

Contribution à l'histoire des traductions juives de la Bible hébraïque

\section{La Bible entre ses langues :de l'hébreu au yiddish}

Michèle Tauber

\section{(2) OpenEdition}

Journals

Édition électronique

URL : https://journals.openedition.org/tsafon/1697

DOI : 10.4000/tsafon. 1697

ISSN : 2609-6420

Éditeur

Association Jean-Marie Delmaire

Édition imprimée

Date de publication : 1 septembre 2019

Pagination : 35-54

ISSN : $1149-6630$

\section{Référence électronique}

Michèle Tauber, «La Bible entre ses langues :de l'hébreu au yiddish », Tsafon [En ligne], 77 | 2019, mis en ligne le 12 septembre 2019, consulté le 23 juin 2021. URL : http://journals.openedition.org/tsafon/ 1697 ; DOI : https://doi.org/10.4000/tsafon. 1697 


\section{La Bible entre ses langues : de l'hébreu au yiddish}

Michèle Tauber*

La Bible juive demeure le fondement sur lequel se construit et se développe la majeure partie de la littérature yiddish ancienne. Elle occupe une place centrale, comme support de l'éducation et de la transmission du judaïsme auprès des masses juives, que ce soit dans les glossaires, les dictionnaires, les concordances, les traductions en vers ou en prose et les commentaires homilétiques, et comme texte à partir duquel se forge une tradition littéraire juive en langue vernaculaire, épopées, chansons de geste ou poèmes et drames bibliques ${ }^{1}$.

\section{Prépondérance du texte biblique dans la vie juive}

À toutes les époques de l'histoire juive, le texte révélé a occupé une place centrale dans la vie religieuse. Le fait qu'il ait été inspiré par Dieu et consigné en hébreu, la langue divine, par les prophètes habités par l'esprit saint, ruah ha-qodesh, ne pouvait que lui conférer un statut à part à l'intérieur du vaste corpus des textes saints. Dans le monde ashkénaze, la prédominance de la Bible reste évidente puisque la lecture, la récitation, l'apprentissage et le commentaire du texte biblique sont au cœur de la vie religieuse juive. Dans le même temps, le lernen, l'étude, occupe une fonction centrale dans la culture ashkénaze : il intervient à tous les âges de la vie et demeure l'occupation exclusive du talmid

\footnotetext{
${ }^{*}$ Université Sorbonne Nouvelle - Paris 3.

${ }^{1}$ Jean Baumgarten, Introduction à la littérature yiddish ancienne, Paris, Les Éditions du Cerf, 1993, p.109.
} 
hakham $^{2}$, dont on sait qu'il jouit d'un immense prestige dans la société juive. L'étude intervient à chaque stade du système éducatif juif comme un des piliers de la transmission. Ce devoir incombe d'abord aux parents qui doivent inciter, dès le plus jeune âge, à l'étude de la Torah, à la récitation de prières, dont le Shema Israël ${ }^{3}$ ou le Birkat Ha-mazon ${ }^{4}$ et au respect des devoirs sociaux dont la charité ou la visite des malades. Au heder, l'école primaire, dès quatre ou cinq ans, l'enfant commence l'initiation aux lettres hébraïques, le déchiffrage et la mémorisation des mots par la lecture de fragments du Lévitique. Le cursus de l'étude aboutit, pour les enfants les plus doués, à la yeshiva, l'école talmudique. La lecture quotidienne de la Bible intervient dans de nombreuses autres situations, surtout à la synagogue, lors de la lecture de la Torah qui s'échelonne durant toute l'année et s'accompagne de la lecture des haftoyres ou passage des Prophètes. La sidrè, ou péricope hebdomadaire, est complétée par son explication lors de la droshe, le sermon rabbinique. La lecture biblique intervient également dans les lieux d'étude individuelle, lernen far zikh, comme le beysmedresh, la maison de prières et d'étude, dans les hevres ${ }^{5}$, confréries saintes ou dans l'espace domestique lors des lectures pieuses d'oyneg shabbes, plaisir du shabbat. La lecture de la Bible et des textes saints imprègne donc tout le quotidien de la vie et scande l'année liturgique juive ${ }^{6}$.

\section{Traduire le texte sacré}

La question de la traduction de la Bible en langue vulgaire s'est posée à de nombreuses époques de l'histoire juive. Cette pratique s'est révélée nécessaire chaque fois que, en raison d'un phénomène de diglossie interne propre à de nombreuses communautés de la diaspora, une frange de fidèles s'est trouvée éloignée de la lecture et de la

\footnotetext{
${ }^{2}$ talmid hakham: (hébreu) : érudit en matières juives qui n’a pas encore été fait rabbin. Il représente ensuite un idéal du judaïsme rabbinique qui exige, outre l'érudition, une personnalité aimable et de nombreux traits éthiques.

${ }^{3}$ Le Shema Israël - litt. : «Écoute Israël »- est le texte principal de la liturgie juive. Composé de trois extraits de la Torah, on le récite matin et soir accompagné de bénédictions.

${ }^{4}$ Birkat Ha-mazon - litt. : « Bénédiction de la nourriture »- est la prière juive récitée après chacun des trois repas.

${ }^{5}$ hevres : on connaît ainsi dans la société ashkénaze des hevres mishnayes, confréries d'étude de la Mishna, des hevres Eyn Yankev, confréries d'étude du Eyn Ya'akov, recueil des parties aggadiques du Talmud.

${ }^{6}$ J. Baumgarten, Introduction, op. cit., p. 116-118.
} 
compréhension du texte saint. Pour ne pas perdre le contact avec la principale source vivante de la tradition du peuple juif, les sages ont toléré que la Bible soit transposée en langue étrangère ou dans la langue juive vernaculaire, mais selon des règles strictes et en conservant toujours son caractère de sainteté au texte révélé.

Après le retour de l'exil de Babylone, autour de -538 , le prophète Néhémie décrit ainsi une scène de récitation publique de la Bible qui unit prière et enseignement :

Et tout le peuple se réunit comme un seul homme sur la place [...] et ils dirent à Ezra le scribe d'apporter le livre de la Loi de Moïse que Dieu avait prescrite à Israël. Ezra le prêtre apporta la Loi en face de l'assemblée [où se trouvaient] les hommes, les femmes et à tous ceux capables de comprendre la lecture. [Puis] : Ils lurent dans le livre de la Loi de Dieu, en l'expliquant, en en donnant le sens et en faisant comprendre la lecture. Néhémie, VIII, 1 - 3 et VIII - 8.

L'emploi de l'expression "à tous ceux capables de comprendre » laisse supposer que l'hébreu était devenu, pour certains, une langue inaccessible, opaque. L'usage s'instaure alors d'accompagner la lecture de l'original de la Bible d'une paraphrase en langue vernaculaire dans le but de rendre le texte révélé compréhensible au plus grand nombre. Dans une explication de ce verset, le Talmud évoque cette coutume :

'Ils lurent dans le livre de la Loi de Moïse' se rapporte au texte hébreu; 'en l'expliquant' signifie 'en traduisant de l'hébreu en araméen' (la langue devenue courante parmi les Juifs de Palestine). Meforash, 'en l'expliquant' vient de la racine hébraïque $P-R-S H$ qui signifie aussi «séparer», «couper». 'En l'expliquant' peut donc vouloir dire «en séparant distinctivement les mots», « en séparant section par section », « en traduisant et en commentant» et «en développant l'interprétation du texte biblique selon la méthode du targum. ${ }^{7}$

L'usage se répand de faire appel, durant l'office à la synagogue, à des meturgemanim, traducteurs officiels chargés de guider, de transposer et d'interpréter la péricope de la semaine. Ces traductions parlées ont été ensuite mises par écrit, ce sont les targumim, à la fois paraphrases et explicitations des versets de la parasha ${ }^{8}$. Toutefois l'éloignement ou l'oubli de la langue sainte nécessitent l'invention de dispositifs nouveaux

\footnotetext{
${ }^{7}$ targum : traduction de la Bible hébraïque en araméen, texte hébreu de la Torah accompagné de commentaires en araméen, langue qui s'était substituée à l'hébreu pendant la captivité des Juifs à Babylone (entre - 586 et - 538).

${ }^{8}$ parasha: synonyme de sidrè, autre terme pour désigner la péricope hebdomadaire lue le shabbat matin à la synagogue.
} 
de transmission, afin de pouvoir diffuser l'essence de la tradition juive au plus grand nombre et de conserver une place centrale à la lecture et à la connaissance du texte révélé ${ }^{9}$.

Ainsi la transmission de la Torah par le biais de la langue vulgaire devient un usage courant dans toutes les communautés de la diaspora. Cette pratique est à l'origine de la quasi-totalité des littératures en langues juives. Les traductions de la Bible en langue vernaculaire ont toujours joué un rôle très important comme rempart contre l'effritement de la tradition et contre l'extension de l'ignorance, et ceci d'autant plus durant les périodes de crise comme celles que traversaient les communautés juives d'Europe à l'époque des temps modernes. Il s'agissait également, en diffusant ces Bibles vernaculaires, de combattre le danger latent d'intellectualisation de la vie religieuse juive et de professionnalisation du savoir qui risquait de rejeter une partie des fidèles dans les marges de la transmission. Les principales littératures juives possèdent toutes des traductions de la Bible, témoignage du souci constant de populariser le message divin, de l'adapter aux multiples koïnè $^{10}$ de la diaspora.

À partir du $\mathrm{XVI}^{\mathrm{e}}$, siècle la langue vernaculaire se trouve au cœur du débat qui agite les communautés juives et qui concerne au premier chef l'instruction religieuse du peuple. Deux aspects cardinaux sont principalement discutés, les traductions de la Bible en yiddish et la vernacularisation de la liturgie. C'est par nécessité plus que par conviction que les traductions de la Bible interviennent, à partir de cette époque, dans la nouvelle stratégie pédagogique qui est mise en place, afin de favoriser une meilleure éducation et une édification des masses juives. La traduction de la Bible en yiddish répond, en effet, à une double démarche : les milieux cultivés voient dans ces traductions une arme efficace pour faire renaître la religiosité du peuple et lutter contre l'inculture religieuse, l'ignorance et mieux encadrer les masses. Les autorités rabbiniques adoptent une position ambivalente, tantôt de rejet pur et simple, tantôt plus conciliante compte tenu de la nécessité de restaurer une connaissance suffisante de la Torah parmi les masses juives. En effet, les traductions yiddish ne remettent-elles pas en cause, certes dans une faible mesure, la suprématie jusqu'alors toujours exercée par les lettrés sur la lecture et le commentaire des textes saints ? De plus ne risque-t-on pas de voir des non-initiés s'arroger des compétences qui

\footnotetext{
${ }^{9}$ J. Baumgarten, Introduction, op. cit., p. 120-121.

${ }^{10}$ Kö̈nè : (grec) : commun. Il s'agit des langues communes à une population donnée.
} 
demeurent jusqu'alors l'apanage exclusif des couches cultivées, gardiennes de l'interprétation et de la diffusion des textes saints? N'y at-il pas danger de déviance hétérodoxe, de travestissement du texte saint et surtout d'interprétations erronées ? Le fait de traduire le texte révélé en langue vulgaire implique donc une lente redéfinition des usages et des habitudes religieuses instituées ${ }^{11}$.

\section{Parallèle avec la Réforme}

La culture de la Réforme qui se diffuse dans les pays germaniques à partir du $\mathrm{XVI}^{\mathrm{e}}$ siècle n'est pas sans avoir laissé de traces sur la littérature juive en langue vernaculaire. On constate un certain nombre de parentés ou similitudes entre les revendications des protestants dans le monde germanique et nombre d'idées qui prévalent chez certains auteurs juifs ou traducteurs de la Bible en yiddish. Ainsi par exemple la plus large place donnée au vernaculaire, comme en témoignent quelques unes des exigences des fondateurs de la Réforme, dont la nécessité de traduire la Bible en langue vulgaire et son rôle accru dans la liturgie et la prédication. Il s'agit non seulement d'un retour de la parole de Dieu pour tous, y compris les chrétiens ordinaires et notamment les femmes, mais encore au texte biblique seul, dans son sens simple, littéral. Le parler quotidien, jusque-là peu utilisé dans le monde de l'Église dominé par le latin, devient un des véhicules grâce auquel se diffuse le message divin, autorité suprême et nourriture spirituelle exclusive de chaque croyant. À un univers religieux et intellectuel largement dominé par le latin, langue universelle de culture, de spéculations théologiques et de l'exégèse biblique, se substitue un nouveau mode de communication marqué par un bilinguisme de fait.

Martin Luther constitue l'exemple le plus représentatif de ces nouvelles donnes culturelles puisque, dans ses textes, il fit un usage combiné de deux langues, le latin et l'allemand. Il écrivit en latin pour les théologiens, les papes ou ses étudiants, et en langue vulgaire lorsqu'il prêchait au peuple des fidèles, aux paysans révoltés ou dans les pamphlets qu'il adressait aux princes des cités germaniques ralliés à la Réforme. Dans le monde catholique, l'exemple le plus probant reste celui de Maître Eckhart ${ }^{12}$ dont les œuvres oscillent, selon les besoins et le

\footnotetext{
${ }^{11} \mathrm{~J}$. Baumgarten, Introduction, op. cit., p. 118-119.

${ }^{12}$ Eckhart von Hochheim, dit Maître Eckhart (c. 1260 - c. 1328) : théologien et philosophe dominicain, le premier des mystiques rhénans.
} 
public visé, entre textes théologiques ou commentaires bibliques en latin et sermons destinés aux simples croyants en langue allemande.

\section{Les premières adaptations en yiddish : glossaires et concordances}

Les adaptations bibliques en yiddish se caractérisent par quelques traits bien particuliers. Les traducteurs yiddish adoptent les principes essentiels d'interprétation et d'exégèse particuliers à la littérature rabbinique tout en introduisant leurs propres modalités. Les Bibles yiddish se caractérisent tout d'abord par un retour au sens littéral du texte saint, au peshat, premier degré d'explicitation du verset. Les auteurs pensent que l'accumulation d'exégèses rabbiniques et talmudiques a travesti et obscurci le texte original qu'il faut rendre à lui-même dans sa pureté et sa clarté. Il s'agit d'avoir un accès direct au texte, débarrassé des ajouts qui éloignent de la source originelle. Voici un passage du Talmud (Shab. 63a) qui pourrait éclairer les intentions des auteurs yiddish :

Rabbi Kahana a dit: "Quand j'avais dix-huit ans je connaissais bien tout le Talmud mais j'ignorais encore qu'un texte ne doit pas sortir de son sens littéral [peshat]». Que devons-nous comprendre? Qu'il faut d'abord avoir une connaissance parfaite [du texte] avant de se livrer à la spéculation.

L'exposition du texte biblique se fait par le recours à la littéralité [mamash], en se servant de la multiplicité des sens contenus dans chaque mot hébreu du texte, ou bien par les correspondances avec d'autres passages bibliques.

La littérature yiddish ancienne reprend ces techniques herméneutiques traditionnelles en les adaptant à un public de lecteurs non initiés dont l'exigence première reste le déchiffrage et la compréhension minimale de la Bible. D'autre part, toute une partie des textes yiddish se fonde sur le deuxième accès essentiel au sens du texte biblique, à savoir le derash, le commentaire proprement dit. Dans cette approche non directement littérale, le commentateur recherche, y compris par des comparaisons en apparence éloignées du texte initial, la signification de tous les détails et la pluralité des sens de l'Écriture sainte, considérée comme une totalité cohérente douée d'une logique interne. C'est dans ce registre d'interprétation, plus libre que le peshat lié à l'explicitation littérale du texte hébraïque, que les auteurs yiddish font intervenir des considérations sur les problèmes contemporains, les tensions religieuses, 
l'impératif éthique et moral destiné à mieux éduquer et contrôler les masses juives. Loin de ne constituer qu'une simple vernacularisation de la tradition sainte, la tradition des Bibles yiddish se révèle riche en renseignements sur les aspirations des lecteurs populaires, ainsi que sur les revendications spirituelles des auteurs yiddish qui ont fait le choix de s'exprimer en langue vulgaire ${ }^{13}$. Cela dit il reste évident que la littérature yiddish ancienne ne pourra jamais se substituer à l'hébreu dans le domaine des commentaires ou des exégèses rabbiniques. Elle ne fera que reprendre, en l'adaptant, une tradition antérieure à laquelle elle s'adossera constamment. Restent exclues également les interprétations kabbalistiques fondées sur le sod, le sens caché du texte biblique. Rédiger un commentaire ésotérique du texte révélé en langue yiddish est contraire aux démarcations entre langue sainte et langue vulgaire. Tout ce qui a trait au domaine halakhique ${ }^{14}$ reste également exclu, à l'exception d'une vulgarisation minimale de la Loi juive, destinée à familiariser le peuple avec les pratiques les plus courantes de la vie juive.

Quels types de textes centrés sur la Bible trouve-t-on alors au sein de la littérature yiddish ancienne ? En premier lieu des glossaires et des concordances qui sont imprimées dès le $\mathrm{XVI}^{\mathrm{e}}$ siècle en Europe. Cette tradition remonte aux gloses yiddish introduites par la formule bileshonenu, en notre langue, ou par bileshon ashkenaz, en langue ashkénaze, (appellations du yiddish ancien que l'on trouve dans les manuscrits et les livres imprimés), gloses qui apparaissent dans les manuscrits médiévaux destinés à expliciter des termes difficiles du texte biblique. Ainsi se constitue un lexique de termes yiddish qui servira de base à la tradition des traductions en langue vernaculaire durant plusieurs siècles ${ }^{15}$.

Grâce à l'imprimerie, cette tradition médiévale des gloses va pouvoir être plus largement diffusée. Parmi cette production il faut citer l'ouvrage anonyme le Mirkeves ha-Mishneh, Le Second Char, titre emprunté à Gn. XLI, 43, allusion aux deux chars qui mènent vers la Torah, l'un en hébreu, l'autre en yiddish. L'ouvrage porte aussi le titre de Seyfer shel rabbi Anshel, Le Livre de rabbi Anshel (Cracovie, 1534) : il s'agit ni plus ni moins du premier livre imprimé connu en yiddish. L'ouvrage se présente sur trois colonnes. Chacune d'entre elles comprend le mot hébraïque en écriture rabbinique carrée, la localisation

\footnotetext{
${ }^{13}$ J. Baumgarten, Introduction, op. cit., p. 122-123.

${ }^{14}$ halakhah : la loi juive.

${ }^{15}$ J. Baumgarten, Introduction, op. cit., p. 123-124.
} 
dans la Bible, plus le ou les équivalents en yiddish ancien en écriture cursive ashkénaze, le vaybertaytsh ${ }^{16}$. À cela s'ajoutent de courtes gloses interprétatives ou des fragments de commentaires, surtout empruntés à Rashi, le commentateur champenois du Moyen Âge, auteur d'un commentaire essentiel de la Bible et du Talmud. Le principe, que l'on retrouvera dans le Humesh-taytsh, la traduction littérale de la Bible, est de conserver dans le yiddish la physionomie de la langue hébraïque. Il s'agit d'un calque de l'hébreu dans lequel à chaque élément morphologique correspond un équivalent yiddish ${ }^{17}$. Sont imprimés également des lexiques dans lesquels les mots sont classés selon leur ordre d'apparition dans le texte biblique. Ainsi le Be'er Moshé, le Puits de Moïse, glossaire du Pentateuque et des Cinq Rouleaux rédigé par Moshé ben Issakhar ha-Levi Sertels et édité à Prague en 1604. L'auteur suggère d'étudier la péricope biblique en entier avant de commencer la suivante et de s'aider de cet ouvrage pour la compréhension des mots hébreux ${ }^{18}$.

\section{Les $\underline{\text { Humoshim-taytsh }}$}

Cette tradition des gloses trouva un prolongement dans le $\underline{\text { Humesh }}$ taytsh, traduction mot à mot du Pentateuque, utilisée essentiellement dans les écoles pour aider au déchiffrage, à la lecture ou à la compréhension du texte saint et éviter toute source d'erreur. On appelle d'ailleurs ces livres hilfsbikher: vade-mecum. Quelques principes essentiels, liés aux méthodes d'enseignement propres aux écoles juives traditionnelles, structurent ces traductions yiddish de la Bible. Ces principes de traduction du texte biblique ne sont pas sans rappeler ceux des targumim. En tout premier lieu, ils se fondent sur la source hébraïque dont ils tendent au maximum à restituer la physionomie, sans forcément tenir compte des règles syntaxiques, morphologiques ou lexicales de la langue parlée. Il s'agit de respecter scrupuleusement la sainteté du texte révélé et donc de conserver en yiddish la physionomie de chaque vocable de la langue sainte. Jamais la suprématie du texte hébraïque ne doit être remise en cause. La langue de traduction se situe entre le langage parlé qui évolue, se transforme, et la langue sainte, canonique, dont le moindre

\footnotetext{
${ }^{16}$ Vaybertaytsh - litt. : yiddish pour les femmes.

${ }^{17}$ J. Baumgarten, Introduction, op. cit., p. 129-130.

${ }^{18} \mathrm{Cf}$. en annexe 1 le début de Be'er Moshé qui montre bien la technique employée dans ces glossaires bibliques : à chaque expression hébraïque - à gauche - correspond une traduction calque en yiddish - à droite.
} 
élément donne du sens. Pour Jean Baumgarten ${ }^{19}$, les traducteurs ont ainsi voulu créer une langue intermédiaire, semi-sacrée, qui n'a ni l'aspect de la langue quotidienne, ni l'intangibilité de la Loi et qui sert principalement pour l'enseignement au heder. C'est une tradition orale transmise par les maîtres, mise par écrit à partir du XIV siècle, puis imprimée.

Il existe par ailleurs de nombreuses versions manuscrites de la Bible en yiddish. Les rédacteurs de ces traductions sont essentiellement des compilateurs qui s'inspirent de versions antérieures et ne font

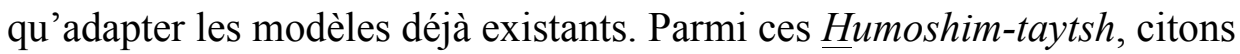
celui traduit par Michael Adam et publié à Constance en 1544. Le compilateur explique d'abord avoir rassemblé, comparé de multiples versions pour rédiger son texte qui s'inscrit dans un ensemble de traductions antérieures. Concernant le mode d'emploi, Michael Adam écrit :

Toute personne qui a peu d'entendement a la possibilité de poser ce livre près d'une Bible en hébreu, esrim ve-arba ${ }^{20}$, et ainsi d'étudier. Tout maître de maison qui ne sait lire que le yiddish [taytsh], peut étudier seul avec son enfant et lui faire comprendre le Humesh. [...] Ce livre est également bon pour les femmes et les jeunes filles qui savent toutes dans l'ensemble bien lire le yiddish, mais passent leur temps dans la lecture de livres sots comme le Dietrich de Berne, Maître Hildebrant ${ }^{21}$ et autres qui ne sont rien que mensonges et choses profanes. Ces femmes et jeunes filles peuvent passer leur temps libre à lire ce Humesh qui n'est que pure vérité. [...] De plus les femmes qui sont à la synagogue et entendent le hazn $^{22}$ lire la sidrè dans le Seyfer toyrè pourront, grâce à ce Humesh en yiddish lire la même sidrè et la haftoyrè et unir leur cœur à celui du hazn.

Le livre saint le plus couramment traduit en yiddish entre le $\mathrm{XVI}^{\mathrm{e}}$ et le $\mathrm{XVIII}^{\mathrm{e}}$ siècle demeure les Psaumes dont la récitation occupe une place centrale dans la liturgie juive. La lecture des Psaumes n'est pas seulement liée à l'office à la synagogue, mais intervient dans de multiples autres situations, que ce soit par exemple lors des lectures pieuses dans les hevres tehilim ${ }^{23}$ ou comme moyen de guérison dans les

\footnotetext{
${ }^{19}$ J. Baumgarten, Introduction, op. cit., p. 133-135.

${ }^{20}$ esrim ve-arba': (hébreu) - litt. : vingt-quatre, c'est-à-dire les Vingt Quatre livres de la Bible hébraïque.

${ }^{21}$ Transcriptions en yiddish des chansons de geste de l'Occident chrétien. L'un des buts de la littérature yiddish ancienne est de lutter contre les influences des lectures profanes. ${ }^{22}$ hazn: (hébreu) : chantre à la synagogue.

${ }^{23}$ hevres tehilim: (hébreu) : confréries saintes dans lesquelles on étudiait et on récitait les Psaumes.
} 
sifrey refu'es ${ }^{24}$. La méthode d'apprentissage du texte biblique au heder est fondée sur deux types d'explication du Humesh. La première consiste à faire l'analyse mot à mot des versets qui sont traduits en vue de la compréhension de leur sens littéral. Cette pratique est à l'origine des versions littérales des livres bibliques qui viennent d'être évoqués. Dans un second temps, le melamed ${ }^{25}$ revient sur le verset en entier pour en saisir la compréhension globale et il ajoute des interprétations puisées dans la littérature talmudique, midrashique ${ }^{26}$ ou dans les commentaires des rabbins médiévaux, dont Rashi. À partir de l'autorité première que constitue le texte biblique, se déploie une multitude d'interprétations visant à interroger et éclairer le message divin. La méthode traditionnelle d'exégèse rabbinique génère un type d'ouvrages dans lequel alternent traductions - fartaytshung - et explications du peshat ou du derash ${ }^{27}$.

\section{La Tsenerene}

L'ouvrage le plus achevé et le plus célèbre de cette tradition reste la traduction intitulée Tse'enah u're'enah, ou Tsenerene, de Ya'aqov ben Yitshok Ashkenazi de Janow (Janow 1550 - Prague 1628), qui a été composé au $\mathrm{XVI}^{\mathrm{e}}$ siècle mais dont la première édition qui nous soit parvenue est celle de Lublin, $1622^{28}$.

L'auteur, originaire de Janow, bourgade des environs de Lublin, était prêcheur itinérant et vraisemblablement marchand de livres. Compte tenu de l'étendue de son érudition, il devait certainement être un savant, versé dans la connaissance de la Torah et des commentaires. Trois de ses œuvres nous sont parvenues: une en hébreu: Shoresh Ya'aqov (Cracovie, 1585), un ensemble de dinim, de lois; et deux en yiddish : le Melitz yoysher (Lublin, 1622), un recueil de commentaires bibliques, et la Tsenerene, commentaire du Pentateuque, des Cinq Rouleaux et des haftoyres, organisé selon la péricope hebdomadaire lue à la synagogue. Cet ouvrage se compose d'une multitude de sources, que ce soit des exégèses de la Torah, des légendes talmudiques et midrashiques, des extraits du Zohar, des commentaires médiévaux, sans oublier des fragments éthiques dans la lignée des muser-sforim, livres de morale, et des considérations sur les coutumes et pratiques liées à la tradition des

\footnotetext{
${ }^{24}$ sifrey refu'es : (hébreu) : livres de médications.

${ }^{25}$ melamed : (hébreu) : enseignant.

${ }^{26}$ De midrash, pluriel midrashim : (hébreu) : commentaire autour du texte biblique.

${ }^{27}$ J. Baumgarten, Introduction, op. cit., p. 141.

${ }^{28}$ Cf. en annexe 2 le frontispice de deux éditions : Nowi Dwor, 1784, et Vilnè, 1895.
} 
minhogim-sforim, livres d'usages. L'ouvrage se présente comme une encyclopédie populaire de la tradition sainte et un guide spirituel et moral à l'usage des lecteurs non initiés. Cette forme anthologique explique qu'il reste le livre yiddish le plus répandu dans les communautés juives ashkénazes. Le titre, emprunté au verset biblique : «Sortez et regardez, filles de Sion » (Ct., III, 11) prouve qu'il s'agit essentiellement d'une Bible pour les femmes, principal public de ce genre d'ouvrages vernaculaires. Dans les foyers juifs, notamment lors des lectures pieuses d'oyneg shabbes, alors que l'homme étudiait une page de Guemara, a blat Gemore, la femme, elle, lisait ou étudiait des ouvrages dont principalement cette Tsenerene. En fait ces ouvrages yiddish semblent s'être adressés à tous ceux pour qui les livres saints restaient des textes " clos par un sceau hermétique ", sefer hatum, faute de compréhension.

À première vue, la Tsenerene ne semble être qu'une adaptation vernaculaire de textes saints à l'usage du peuple juif. On peut se demander alors pour quelle raison il est considéré comme un ouvrage classique et où se situe son originalité à l'intérieur de la littérature juive $^{29}$. Il a tout d'abord une importance historique majeure du fait qu'il marque une rupture dans la méthode de transmission et d'accès au texte biblique et témoigne d'une certaine libération par rapport à la tradition des Humoshim-taytsh. Il ne s'agit pas d'une traduction mécanique mais d'une libre adaptation de la Torah entremêlée de sources très variées elles-mêmes réécrites.

Il y a une sorte de mutation [écrit Jean Baumgarten], marquée par le passage de l'ouvrage didactique utilisé par le maître pour enseigner la Bible aux enfants, au livre de lecture, caractérisée par une narration vivante des principaux épisodes bibliques [... le livre] permet de comprendre comment s'opèrent la circulation dynamique et les échanges complexes entre l'univers des savants et le monde des simples lecteurs. ${ }^{30}$

On peut, comme cela a été le cas durant de nombreux siècles, ne voir dans cette production qu'un reflet édulcoré des sources originales, une banale popularisation des textes hébraïques qui seraient descendus des cercles initiés au public populaire, une sorte de sous-produit de la haute culture juive. On élude alors le problème de la composition de ces ouvrages, on ne cherche pas à comprendre comment sont traitées les

\footnotetext{
${ }^{29}$ J. Baumgarten, Introduction, op. cit., p. 145-146.

${ }^{30}$ Ibid., p.146-147.
} 
sources premières, de quelle façon sont recomposés, agencés les textes canoniques qui tissent la trame de ce livre.

Il ne s'agit jamais dans la Tsenerene d'une soumission passive à des modèles forgés par les lettrés ou d'une simple adaptation édulcorée mais bel et bien d'une réécriture à l'usage des non-initiés, ce qui suppose une authentique création, la parfaite maitrise des sources originales, l'art de faire passer en langue vulgaire la richesse et la profusion des commentaires, une habileté à rendre clairs ou à expliciter dans un style vivant les multiples sens parfois hermétiques de la Torah. $^{31}$

L'originalité se manifeste en outre dans le choix des commentateurs: Ya'aqov ben Yitshok Ashkenazi de Janow aime à privilégier les passages les plus utiles du Midrash rabba ou de Rashi, mais aussi des explications moins courantes qui n'en prennent que plus de relief. Rappelons que l'auteur est un prêcheur itinérant : fonction qui jette à la fois une lumière sur l'origine sociale et culturelle de nombreux auteurs de cette littérature yiddish, sur la façon dont s'organise le texte et sur la circulation entre culture en hébreu et culture en langue vernaculaire. Le prêcheur possède en effet le savoir des savants sans toutefois avoir obligatoirement reçu une reconnaissance officielle de la part des notables ou des rabbins. Il occupe une situation intermédiaire puisqu'il est issu du monde de l'étude, mais qu'il peut être également en rupture de ban, voire en opposition avec ceux qui incarnent l'autorité. D'où sa vie errante au contact du peuple juif et sa compréhension des besoins et des aspirations populaires. Sa position médiane en fait le diffuseur privilégié de la tradition auprès de ceux qui ont peu le temps et l'opportunité d'étudier. Le prêcheur est l'un des canaux essentiels par lequel circule et se réécrit, en langue vernaculaire, le savoir religieux ${ }^{32}$.

L'ouvrage s'apparente aux sermons en relation avec la parasha, qui étaient délivrés à la synagogue durant l'office ou les fêtes afin d'inculquer les règles de vie, les enseignements moraux, la diffusion de la Loi et le respect des commandements. L'agencement du texte correspond à un style bien codifié : l'explication commence par une paraphrase en langue vulgaire du verset: fartaytshung, suivie d'une introduction, petiha, composée le plus souvent d'une citation extraite d'un livre prophétique, souvent aussi des Proverbes ou des Psaumes. Suivent des paraboles, histoires, légendes talmudiques ou commentaires

\footnotetext{
${ }^{31} I d$.

${ }^{32}$ Ibid., p. 149.
} 
midrashiques qui permettent d'arriver à l'explication proprement dite du verset de la parasha. Dans cette exégèse se mêlent des extraits significatifs de commentaires, des passages abrégés de Midrashim, des propos édifiants et un rappel des pratiques juives. Ces passages se terminent parfois par une prière ou une invocation pour la rédemption du peuple d'Israël et le repentir des Juifs. Dans le commentaire suivi de chaque verset, l'auteur utilise l'explication littérale des mots difficiles ou des notions essentielles, le recours au questionnement suivi de réponses inspirées par les principaux commentateurs. La narration moralisante prend le pas sur l'exposé théologique lié au pilpul, raisonnement talmudique, dont il se démarque visiblement. L'auteur montre un certain goût pour les dialogues, la dramatisation des scènes, afin de précipiter l'envie de repentir et de capter l'attention des lecteurs. Les explications sont marquées par une constante allégorisation. La narration qui permet d'introduire l'enseignement de la Torah à travers la fiction, la légende aggadique $^{33}$ ou la parabole talmudique, induit des préceptes moraux et donne accès à la Loi et à l'éthique ${ }^{34}$.

L'extrait en annexe 3 - Gn, XII, la célèbre injonction biblique faite à Abraham - montre bien la méthode d'écriture de Ya'aqov ben Yitshok Ashkenazi de Janow : il puise dans la vaste tradition des interprétations rabbiniques qui servent de trame à son propre commentaire, en introduisant toutefois des changements, notamment la brièveté et la concision. Le style oscille entre le respect des formes propres à la littérature midrashique et l'invention d'une langue qu'on peut déjà qualifier de «littéraire ${ }^{35}$. La richesse de ce texte réside dans la constante tension entre d'un côté la pensée la plus orthodoxe destinée à familiariser le peuple avec les sources traditionnelles et de l'autre les savoirs et les pratiques populaires. Dans de multiples chapitres du livre transparaissent des représentations et des croyances qui rendent compte de modes de vie ou d'une sociabilité propres au peuple juif. On peut alors mieux comprendre l'existence quotidienne, les souffrances de l'exil et l'espérance messianique des masses juives d'Europe. L'auteur peut insister ainsi tout à la fois sur l'impératif de la teshuva, du repentir, dresser un catalogue des délits et des fautes qui retardent la rédemption,

\footnotetext{
${ }^{33}$ De aggada, pluriel aggadot: (hébreu) : commentaire et légendes autour du texte biblique.

${ }^{34} \mathrm{~J}$. Baumgarten, Introduction, op. cit., p. 149.

${ }^{35} \mathrm{Il}$ faut à ce sujet rappeler l'importance que la Tsenerene aura en tant que source lexicale pour des écrivains yiddish modernes, et notamment le premier d'entre eux Mendele Moykher Sforim (1836 - 1917), fondateur de la littérature yiddish moderne.
} 
mettre en garde contre la débauche et les superstitions fustigées comme d'intolérables déviances, et présenter un tableau vivant de pratiques magiques, de préjugés ou de croyances liés aux grands moments de la vie ou en relation avec les fêtes domestiques.

La représentation de la femme juive qui est donnée dans le livre fournit un bon exemple de ces tensions dramatiques qui caractérisent l'œuvre. La femme occupe une place centrale, comme pilier de la transmission, de l'éducation et comme cœur vivant du foyer juif. Elle est magnifiée à l'égal des matriarches, comme génitrice et dispensatrice d'une multitude de savoirs. En cela la Tsenerene traduit une évidente promotion de la femme au sein de la société juive. Longtemps laissée dans les marges de la littérature religieuse juive, elle y fait irruption comme figure centrale, aussi bien dans la vie quotidienne que comme élément moteur du processus de rédemption messianique. Mais la femme est tout autant rabaissée, voire condamnée, parce que source de péché et de transgression. La tradition magique est également présente dans le texte comme en témoignent les multiples allusions aux pratiques démonologiques qui émaillent le texte, mais visiblement pour mieux être exorcisée et dénoncée.

À noter également la place essentielle du corps humain, lieu par excellence de conflits, d'un écartèlement constant entre ordre et turbulence. La maladie, la mort, la sexualité, la grossesse et l'accouchement sont souvent évoqués, tout autant que la félicité du corps dans l'autre monde. On discerne un heurt continuel entre puritanisme et évocation du penchant au mal, yetser hore, entre tendances rationnelles et superstitions, entre religion instituée et déviances populaires. Autant d'oppositions qui font de ce livre le lieu des contradictions extrêmes du judaïsme et le réceptacle de croyances éclatées, contradictoires, bien représentatives d'aspects fondamentaux de la pensée juive de l'époque des temps modernes.

L'auteur, en réintégrant dans l'édifice culturel une frange rejetée de la société juive, manifeste sa volonté de fonder une nouvelle cohésion audelà des déchirures sociales et religieuses. Cette visée unificatrice se révèle d'autant plus importante dans cette époque $\left(\mathrm{XVI}^{\mathrm{e}}\right.$ et $\mathrm{XVII}$ siècles), marquée par des violences antijuives, des persécutions, des conversions forcées et des crises internes qui menacent la survie des communautés $^{36}$. Ainsi transparait la portée historique de cette littérature en yiddish, non seulement espace d'énonciation de la culture des Juifs

\footnotetext{
${ }^{36}$ J. Baumgarten, Introduction, op. cit., p. 151-152.
} 
ordinaires mais encore réponse des doctes face aux dangers de désintégration de la vie juive. On comprend alors pourquoi la Tsenerene a pu être un viatique pour tant de générations juives gagnées par l'ignorance ou le désespoir et pourquoi elle connut une telle célébrité dans le monde yiddishophone. Le caractère exemplaire de ce livre réside aussi dans l'exceptionnelle longévité de son usage : la première édition est celle de Hanau (Bâle, 1622). La page de titre mentionne trois éditions antérieures (Lublin, 1615 ; deux à Cracovie 1618, 1620). Au XVII ${ }^{\mathrm{e}}$ siècle ont été imprimées plus de vingt éditions dans les principaux centres d'édition hébraïques en Europe: Prague, Amsterdam, Cracovie, Francfort-sur-le-Main, et Francfort-sur-Oder, Sulzbach. Du XVII ${ }^{\mathrm{e}}$ au $\mathrm{XX}^{\mathrm{e}}$ siècle se sont succédé plus de deux cent dix éditions de la Tsenerene. Au XX ${ }^{\mathrm{e}}$ siècle, le livre suit les migrations juives : États-Unis, Argentine, Israël. À partir de la Haskala ${ }^{37}$, le texte subit diverses évolutions. Les maskilim ${ }^{38} \mathrm{du} \mathrm{XIX}^{\mathrm{e}}$ siècle adaptent la langue et rajeunissent le texte en supprimant les références trop superstitieuses, les allusions aux pratiques magiques ou aux croyances jugées trop désuètes perçues comme des obstacles à l'émancipation des lecteurs juifs. Autre indice de la popularité de la Tsenerene : l'abondance des adaptations et des traductions : traduction en latin des chapitres 1 à 5 de la Genèse en 1660 par J. Saubert, puis dans les principales langues européennes ${ }^{39}$.

\section{L'après Tsenerene}

Si l'on observe la longue suite de traductions de la Bible en yiddish, on constate que la première version intégrale de la Bible juive en langue vulgaire n'a été imprimée qu'au XVII siècle à Amsterdam. Il s'agit des Torah, nevi'im, ketuvim bileshon ashkenaz ${ }^{40}$, traduits par Yequti'el ben Yitshoq Blitz (1676-1679), éditeur Uri Phoebus ben Aharon ha-Levi), et par Yossef ben Alexander Witzenhausen chez Joseph Athias (1679). Les deux traducteurs rejettent les exégèses rabbiniques et talmudiques, selon eux ces accumulations d'interprétations obscurcissent et travestissent le texte original qu'il faut rendre à luimême dans sa pureté et sa clarté. Deux principes guident leur travail : le retour au sens littéral, peshat, et le refus de sens explicatif, derash. Ils

\footnotetext{
${ }^{37}$ Haskala : (hébreu) : mouvement des Lumières juives (1780-1880).

${ }^{38}$ Maskilim : (hébreu) : tenants de la Haskala.

${ }^{39}$ J. Baumgarten, Introduction, op. cit., p. 153-154.

${ }^{40}$ Torah, nevi'im, ketuvim bileshon ashkenaz : (hébreu) : Le Pentateuque, les Prophètes, les textes hagiograhiques en langue ashkénaze, c'est-à-dire yiddish.
} 
critiquent les traductions-calques qui font obstacle à l'intelligence du texte biblique. L'un de textes les plus fustigés est bien évidemment la Tsenerene dans laquelle le texte biblique est enveloppé dans une multitude de commentaires périphériques. Blitz écrit au sujet de ce livre :

Les gens ordinaires ne peuvent pas étudier ce qui est nécessaire grâce à cet ouvrage car ce ne sont que des midrashim et des aggadot. On pose de nombreuses questions au sujet de la parasha dont on donne les réponses d'après les commentaires et les sermons. Mais jamais, au grand jamais on ne fournit le sens littéral du verset. Cela n'est d'aucune utilité pour les simples lecteurs car le peshat est commenté en fonction du derash et de ce fait on mélange les deux niveaux de sens. ${ }^{41}$

Les auteurs de ces deux traductions remettent en cause les méthodes d'enseignement en vigueur dans les écoles juives d'Europe et leur reprochent d'aboutir à une quasi-méconnaissance de la Bible. L'influence des pratiques d'enseignement en usage dans les écoles d'Amsterdam est évidente et on peut déceler dans les traductions yiddish d'Amsterdam l'effet positif des méthodes pratiquées dans les écoles sépharades de cette ville. Blitz écrit: «J'ai pu observer dans la communauté des Sépharades que la plupart sont de bons connaisseurs de la Bible depuis les petits jusqu'aux plus grands les Sépharades n'ont point leur pareil dans l'étude des versets $\gg{ }^{42}$. Quant à Joseph Athias, lorsqu'il hésitait pour traduire certains passages difficiles, il se rendait dans les batey midroshim ${ }^{43}$ des Sépharades, « grands maîtres de la Torah et fort versés dans l'étude de la Bible ${ }^{44}$, afin de les questionner et d'introduire le minimum d'erreurs dans sa version en yiddish. Les deux traducteurs s'accordent également pour stigmatiser les défauts majeurs des melamdim dans les hadorim $^{45}$ : la primauté accordée au Talmud au détriment du texte biblique. Dès leur plus jeune âge, les enfants sont confrontés à des textes d'une trop grande complexité. L'enseignement est fondé sur la récitation par cœur et la mémorisation, au détriment des règles grammaticales, d'où une connaissance incertaine de l'hébreu et une compréhension illusoire des textes. Uri Phoebus écrit à ce sujet :

Le maître n'a d'autre but que d'apprendre la Guemara au jeune élève. [...] On commence l'apprentissage de l'enfant à l'envers : avant même qu'il sache prier,

\footnotetext{
${ }^{41}$ J. Baumgarten, Introduction, op. cit., p. 158-159.

${ }^{42}$ Cité par J. Baumgarten, ibid, p. 159.

${ }^{43}$ batey midrashim : hébreu, pluriel de beysmedresh : maisons de prière et d'étude.

${ }^{44}$ Cité par J. Baumgarten, Introduction, op. cit., p. 159.

${ }^{45}$ hadorim : pluriel hébreu de heder : école maternelle et primaire.
} 
on commence le Pentateuque, puis on passe trop vite aux mishnayot ${ }^{46}$. Mais compte tenu du développement de son intelligence, il n'y comprend goutte. L'enfant gazouille comme un oiseau mais il ne saisit pas ce qu'il gazouille. ${ }^{47}$

Les deux traductions en yiddish d'Amsterdam marquent une transformation radicale dans les modes d'accès à la tradition : la Bible dans son sens obvie est pour ainsi dire réhabilitée. Elle redevient le texte central de l'étude, de la transmission, de l'apprentissage. La lecture de ces deux Bibles au delà des variantes lexicales ou morphologiques donne une impression de cohérence stylistique et d'unité. Elles ont eu un rôle fondateur à la fois dans la mise à distance des versions anciennes, jugées obsolètes, et dans la transformation des pratiques de traduction du texte biblique. En cela elles annoncent déjà les formes littéraires modernes dont celles des écrivains de la Haskala. Autre influence, cette fois négative: c'est contre ces deux traductions de la Bible que Moses Mendelssohn entreprend en réaction sa propre traduction en nouveau Hochdeutsch. Si ces deux œuvres de Blitz et de Witzenhausen n'ont guère eu de retentissement à leur époque, elles n'en prouvent pas moins que la traduction en langue vernaculaire a été un terrain privilégié d'expérimentation, de renouveau des modes de transmission de la tradition biblique. En cela elles ont joué un rôle évident dans l'évolution de la langue et de la littérature yiddish entre l'époque ancienne et moderne ${ }^{48}$.

\footnotetext{
${ }^{46}$ mishnayot: (pluriel hébreu) : premiers commentaires de la Bible.

${ }^{47}$ Cité par J. Baumgarten, Introduction, op. cit., p. 159.

${ }^{48}$ Ibid., p. 160-161.
} 
ANNEXE 1 : Début de Be'er Moshé qui montre bien la technique employée dans ces glossaires bibliques : à chaque expression hébraïque à gauche - correspond une traduction calque en yiddish - à droite.

$\begin{array}{ll}\begin{array}{l}\text { bereshit } \\ \text { bara }\end{array} & \begin{array}{l}\text { in ershtenish odr am an fang } \\ \text { tohu vabohu }\end{array} \\ \text { tehum } & \text { veshefenish } \\ \text { veruah ler } & \text { apgrunt } \\ \text { merahefet } & \text { gemit odr nevua } \\ \text { yehi rakya' } & \text { shvebt-er makht shvebn } \\ \text { betokh } & \text { es zol } \text { zikh shterken ein } \\ \text { mayim lamaym } & \text { in tsvishn-in mitn } \\ \text { ykavu } & \text { der intrshten vaser tsu der ibrshten vaser } \\ \text { hamaym } & \text { zi zoln zikh samlen } \\ \text { tadse' } & \text { mer } \\ \text { ose } & \text { zi zol grosen } \\ \text { pri } & \text { vaksung-makht } \\ \text { leminehu } & \text { frukht-ops } \\ & \text { tsu zeyner lei }\end{array}$

ANNEXE 2 : Frontispice de deux éditions de Tsenerene: Nowy Dwor 1784 (à droite) et Vilnè ou Vilnius 1895 (à gauche).

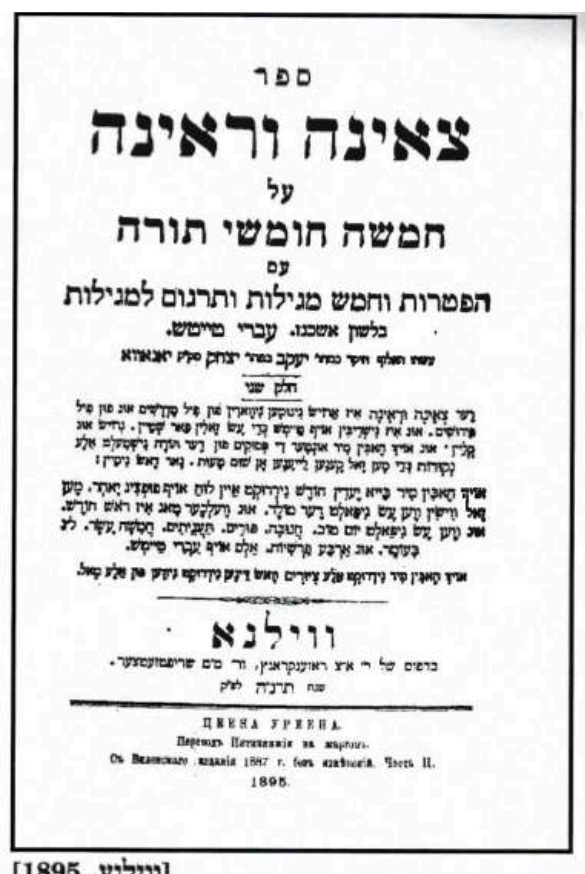

[ורילנע, 1895]

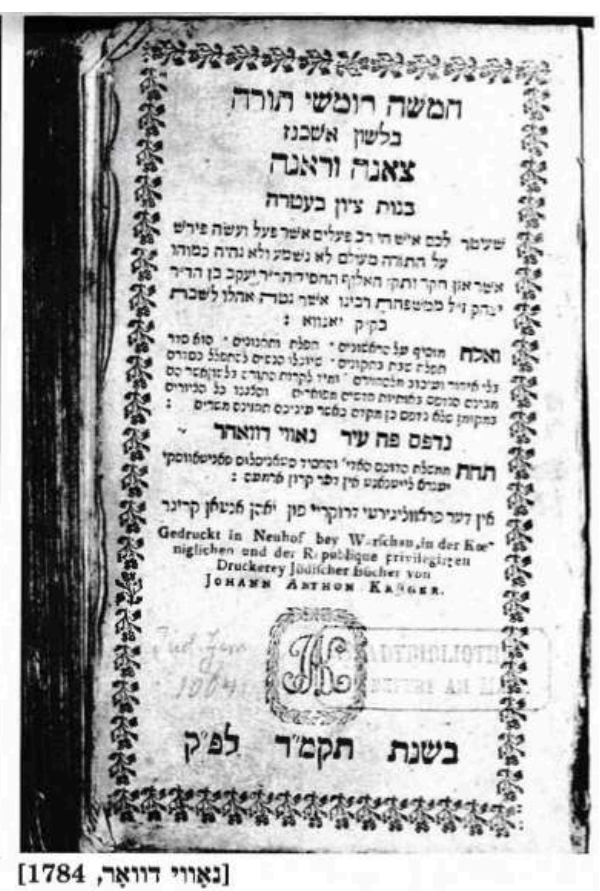

52 
ANNEXE 3 : Tsenerene (yiddish): début de la péricope Lekh lekha, Genèse XII.

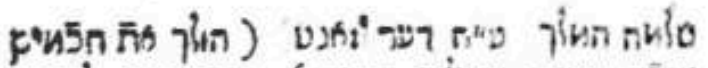

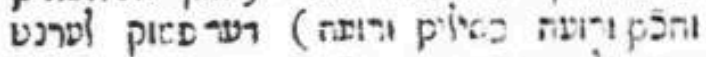

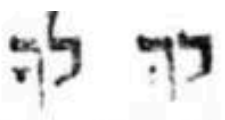

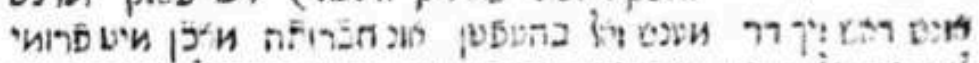

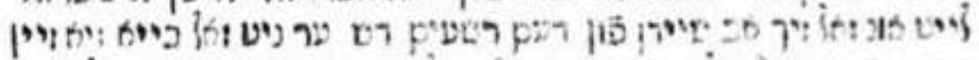

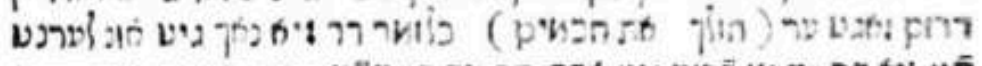

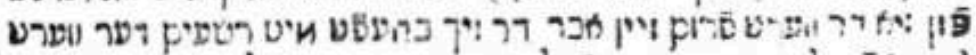

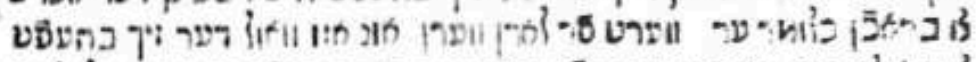

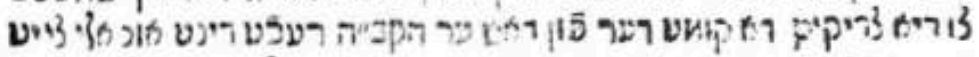

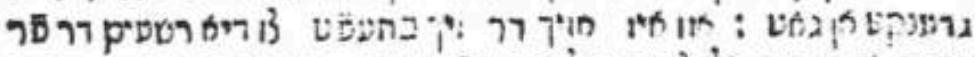

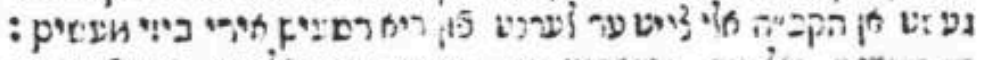
ד רטים

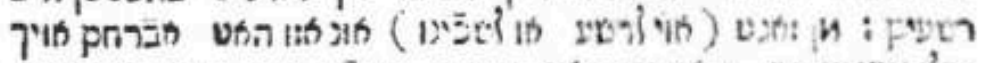

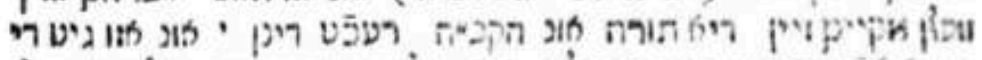

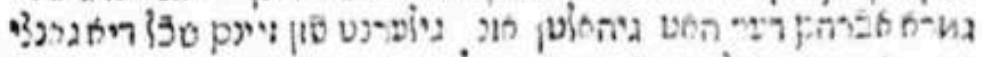

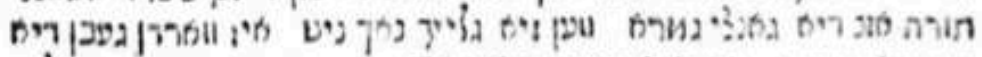

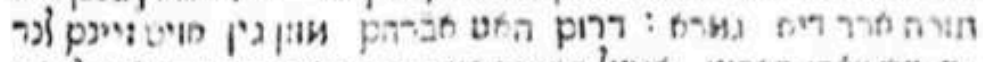

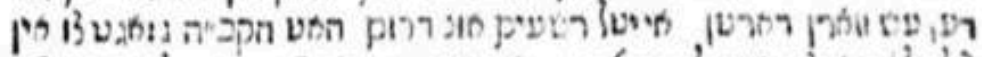

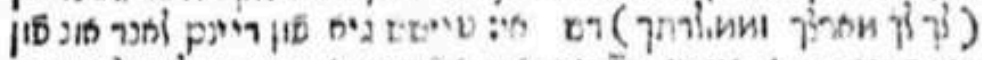

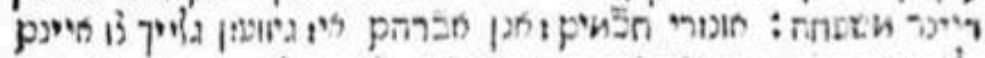
ן מ

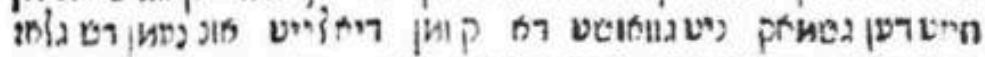

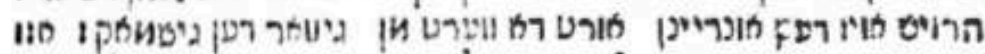

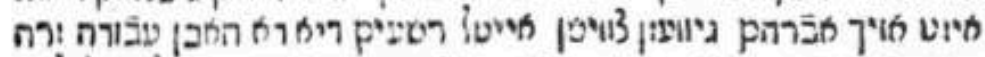

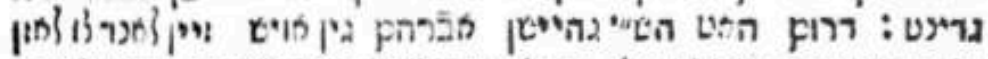

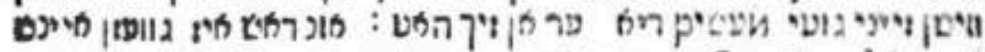

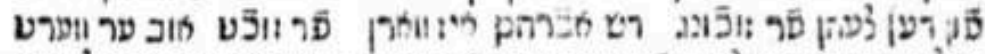

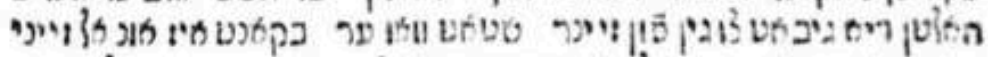

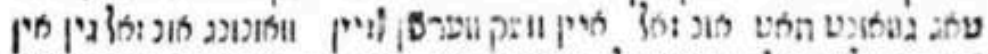

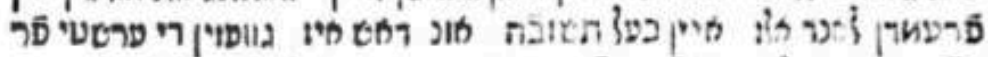

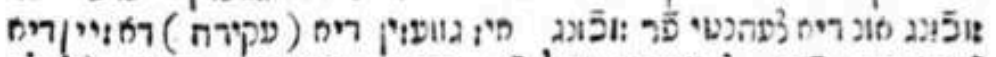

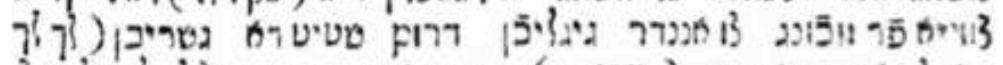

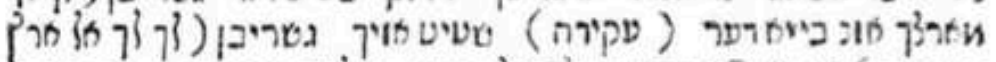
המוריח (

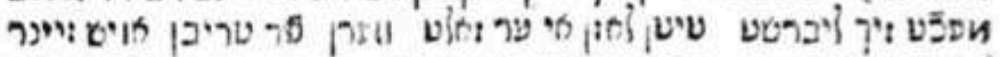

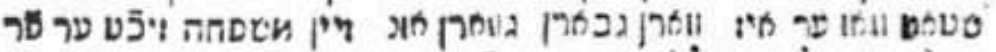
:

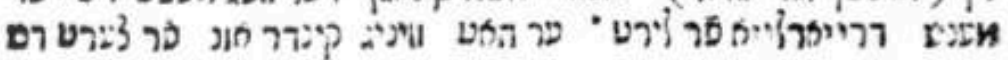


ANNEXE 4 : Traduction du passage de Tsenerene, Genèse XII par Jean Baumgarten, Le commentaire sur la Torah - Tseenah ureenah, Paris, Éditions Verdier-poche, 1987, p. 98.

\section{LECTION LEKH-LEKHA}

VA-T'EN DE LA TERRE (Gen. 12:1). Le roi Salomon, la paix soit sur lui, dit : "Celui qui fréquente les sages devient sage, mais celui qui se plaît avec les insensés s'en trouve mal' ${ }^{1}$. " Ce verset nous apprend que l'homme doit se lier avec des gens pieux et rechercher leur compagnie ; il doit se séparer des impies, éviter de les côtoyer ; c'est pourquoi il est dit : Fréquente les sages, c'est-àdire que l'on doit les suivre et apprendre à devenir pieux à leur contact. Celui qui s'unit avec les impies sera brisé et se perdra. Une personne qui se mêle aux justes est amenée à bien servir le Saint, béni soit-II, à penser continuellement à Lui ; à l'inverse, celui qui s'acoquine avec les méchants, oublie toujours le Saint, béni soit-Il, et apprend de mauvaises actions à leur contact. L'homme doit donc faire très attention de ne pas fréquenter les impies. Ne dit-on pas : Malheur à l'impie, malheur à son voisin² Abraham voulut observer la Torah et bien servir le Saint, béni soit-Il. La Guemara dit ${ }^{3}$ : Abraham observa et étudia par luimême, avec sa seule intelligence, la Torah et la Guemara avant qu'elles aient été données. Abraham dut quitter son pays car de très nombreux impies y vivaient ; c'est la raison pour laquelle le Saint, béni soit-Il, lui dit : "Va-t'en de ta terre, de ta patrie ", ce qui signifie quitte ton pays et ta famille. Nos sages disent : Abraham ressemblait à un verre plein d'épices odorantes qui se

1. Pro. 13:20.

2. Neg. $12 \mathrm{~b}$.

3. Yom. 28b, Ned. 23a, Kid. 4.14. 